Pacific Journal of Mathematic 


\title{
LOCALLY HOLOMORPHIC SETS AND THE LEVI FORM
}

\section{R. HUNT}

\begin{abstract}
Suppose we have a real $k$-dimensional $\mathscr{C}^{2}$ manifold $M$ embedded in $C^{n}$. If $M$ has a nondegenerate complex tangent bundle of positive rank at some point $p \in M$, then the vanishing or nonvanishing of the Levi form on $M$ near $p$ determines whether or not $M$ is locally holomorphic at $p$. We show that if $M$ is locally holomorphic at $p$, then the Levi form vanishes near $p$, the converse being a known result. In addition we prove a $C-R$ extendibility theorem for a certain case when $M$ is $\mathscr{C}^{\infty}$ and has a nonzero Levi form at $p \in M$.
\end{abstract}

1. Introduction. In the study of holomorphic extendibility and holomorphic convexity we often want to know whether a set is a holomorphic set or not. For instance a totally real submanifold of a Stein manifold is a holomorphic set (see [5]). If a real $k$-dimensional $\mathscr{C}^{2}$ manifold $M$ is embedded in $C^{n}$ in such a way that $M$ has a nondegenerate complex tangent bundle at some point $p$, the property of being locally holomorphic at $p$ depends on the Levi form on $M$ near $p$. It has been shown that if the Levi form vanishes near $p$ then $M$ is locally holomorphic at $p$. The converse has been proved only in the generic case when $k>n$ and $M$ is $\mathscr{C}^{\infty}$ ([1] or [6]). It is the purpose of this paper to prove the converse in all cases. For a particular case (which we call pseudo-hypersurface) we combine a lemma of Nirenberg [4] with the compactly supported solutions to the $C-R$ equations to prove a $C-R$ extension theorem.

In $\S 2$ we define exceptional points, the Levi form, and the concept of local holomorphicity. Section 3 contains a discussion of the relation of the Levi form to the local equations of the embedded manifold. In $\S 4$ we show that to prove theorems about local holomorphicity, we need only consider open sets of $C^{n}$ with $\mathscr{C}^{\infty}$ boundaries. We show that a locally holomorphic set has a vanishing Levi form, if the Levi form can be defined. In $\$ 5$ we define the concept of a pseudohypersurface and prove that if the Levi form does not vanish on a pseudo-hypersurface, all $C-R$ functions are extendible to an open set in $C^{n}$.

2. Definitions. Let $M$ be a real $k$-dimensional $\mathscr{C}^{2}$ manifold embedded in $C^{n}, k, n \geqq 2$. Let $T_{x}(M)$ be the real tangent space to $M$ at $x$ and $H_{x}(M)=T_{x}(M) \cap i T_{x}(M)$. Then $H_{x}(M)$ is the maximal complex subspace of $C^{n}$ contained in $T_{x}(M)$, called the vector space 
of holomorphic tangent vectors to $M$ at $x$. It is well known that

$$
\max (k-n, 0) \leqq \operatorname{dim}_{C} H_{x}(M) \leqq\left[\frac{k}{2}\right] .
$$

The real tangent bundle of $M$ is denoted by $T(M)$.

If $f$ is the embedding of $M$ into $C^{n}$, then $J(f)$ denotes the complex Jacobian of $f$ (as a $C$-linear map from $T(M) \otimes C \rightarrow C^{n}$ ). If $q=\min (n, k)$, a point $p$ in $M$ is said to be an exceptional point of order $l, 0 \leqq l \leqq[k / 2]-\max (k-n, 0)$ if the complex rank of $\left.J(F)\right|_{p}$ is equal to $q-l$.

A point $p$ in $M$ is generic if $p$ is an exceptional point of order 0 . The manifold $M$ is locally generic at $p$ if every point in some open neighborhood of $p$ is generic, and is locally $C-R$ at $p$ if every point in some open neighborhood of $p$ is an exceptional point of the same order.

Suppose $M$ is locally $C-R$ at $p \in M$ and $H_{p}(M)$ is nonzero. Then we define the Levi form at any $x$ near $p$

$$
L_{x}(M): H_{x}(M) \longrightarrow \frac{T_{x}(M) \otimes C}{H_{x}(M) \otimes C}
$$

by $L_{x}(M)(t)=\pi_{x}\{[Y, \bar{Y}]\}_{x}$, where $Y$ is a local section of the fiber bundle $H(M)$ (with fiber $H_{x}(M)$ ) such that $Y_{x}=t,[Y, \bar{Y}]_{x}$ is the Lie bracket evaluated at $x$, and

$$
\pi_{x}: T_{x}(M) \otimes C \longrightarrow \frac{T_{x}(M) \otimes C}{H_{x}(M) \otimes C}
$$

is the projection.

A compact set $K$ in a complex manifold $X$ is a holomorphic set (also called a $S_{\delta}$ set) if there is a sequence of open Stein manifolds $X_{i} \subset X$ such that $X_{i+1} \subset X_{i}$ and

$$
K=\bigcap_{i=1}^{\infty} X_{i}
$$

A set $K$ is locally holomorphic at $p \in K$ if there exists a compact neighborhood $N$ of $p$ such that $N \cap K$ is holomorphic.

3. Local equations and the Levi form. Again let $M$ be a real $k$-dimensional $\mathscr{C}^{2}$ manifold embedded in $C^{n}, k, n \geqq 2$. Suppose $M$ is locally $C-R$ at $p$ and $p$ is an exceptional point of order $l$. If $k>$ $n$ the local equations of $M$ in a neighborhood of $p$ are (after a suitable coordinate change) 
(1)

$$
\begin{aligned}
& z_{1}=x_{1}+i h_{1}\left(x_{1}, \cdots, x_{2(n-l)-k}, w_{1}, \cdots, w_{k-n+l}\right) \\
& \vdots \\
& z_{2(n-l)-k}=x_{2(n-l)-k}+i h_{2(n-l)-k}\left(x_{1}, \cdots, x_{2(n-l)-k}, w_{1}, \cdots, w_{k-n+l}\right) \\
& z_{2(n-l)-k+1}=u_{1}+i v_{1}=w_{1} \\
& \vdots \\
& z_{n-l}=u_{k-n+l}+i v_{k-n+l}=w_{k-n+l} \\
& z_{n-l+1}=g_{1}\left(x_{1}, \cdots, x_{2(n-l)-k}, w_{1}, \cdots, w_{k-n+l}\right) \\
& \vdots \\
& z_{n}=g_{l}\left(x_{1}, \cdots, x_{2(n-l)-k}, w_{1}, \cdots, w_{k-n+l}\right),
\end{aligned}
$$

where $x_{1}, \cdots, x_{2(n-l)-k}, u_{1}, v_{1}, \cdots, u_{k-n+l}, v_{k-n+l}$ are local coordinates for $M$ in a neighborhood of $p$ vanishing at $p$, and $z_{1}, \cdots, z_{n}$ are coordinates for $C^{n}$ vanishing at $p$. The real-valued functions $h_{1}, \cdots h_{2(n-l)-k}$ as well as the complex-valued functions $g_{1}, \cdots, g_{l}$ vanish to order 2 at $p$. Because $M$ is locally $C-R$ at $p$, the functions $g_{1}, \cdots, g_{l}$ must be complex-analytic functions of $w_{1}, \cdots, w_{k-n+l}$ (see [3]).

Letting $g_{j}=g_{j}^{\prime}+i g_{j}^{\prime \prime}, j=1, \cdots l$, we find in [7] that the Levi form vanishes at $p$ if and only if the complex Hessians at $p$ of each of the functions $h_{1}, \cdots, h_{2(n-l)-k}, g_{1}^{\prime}, g_{1}^{\prime \prime}, \cdots, g_{l}^{\prime}, g_{l}^{\prime \prime}$ with respect to the variables $w_{1}, \cdots w_{k-n+l}$ all have zero eigenvalues.

Fix $x_{1}, \cdots, x_{2(n-l)-k}$ and expand each $g_{j}$ in a Taylor series in $w_{1}$, $\cdots, w_{k-n+l^{\prime}}$

$$
g_{j}=\sum_{\alpha} a_{j, \alpha} w^{\alpha},
$$

where $w=\left(w_{1}, \cdots, w_{k-n+l}\right)$ and $\alpha=\left(\alpha_{1}, \cdots, \alpha_{k-n+l}\right)$. Replacing $z_{n-l+j}$ by $z_{n-l+j}-\sum_{\alpha} a_{j, \alpha} w^{\alpha}$, we have that $z_{n-l+1}=0, \cdots, z_{n}=0$ in our new local equations. Thus the Levi form vanishes at $p$ if and only if the complex Hessians at $p$ of each of the functions $h_{1}, \cdots, h_{2(n-l)-k}$ are all zero matrices.

If $k \leqq n$ the local equations of $M$ in a neighborhood of $p$ are (after a suitable coordinate change)

$$
\begin{aligned}
& z_{1}=x_{1}+i h_{1}\left(x_{1}, \cdots, x_{k-2 l}, w_{1}, \cdots, w_{l}\right) \\
& \vdots \\
& z_{k-2 l}=x_{k-2 l}+i h_{k-2 l}\left(x_{1}, \cdots, x_{k-2 l}, w_{1}, \cdots, w_{l}\right) \\
& z_{k-2 l+1}=u_{1}+i v_{1}=w_{1} \\
& \vdots \\
& z_{k-l}=u_{l}+i v_{l}=w_{l} \\
& z_{k-l+1}=g_{1}\left(x_{1}, \cdots, x_{k-2 l}, w_{1}, \cdots, w_{l}\right) \\
& \vdots \\
& z_{n}=g_{n-k+l}\left(x_{1}, \cdots, x_{k-2 l}, w_{1}, \cdots, w_{l}\right),
\end{aligned}
$$


where $x_{1}, \cdots, x_{k-2 l}, u_{1}, v_{1}, \cdots, u_{l}, v_{l}$ are the local coordinates for $M$ in a neighborhood of $p$ vanishing at $p$, and $z_{1}, \cdots, z_{n}$ are coordinates for $C^{n}$ vanishing at $p$. The real-valued functions $h_{1}, \cdots, h_{k-2 l}$ as well as the complex-valued functions $g_{1}, \cdots, g_{n-k+l}$ vanish to order 2 at $p$. Since we are assuming $M$ is locally $C-R$ at $p$, the functions $g_{1}, \cdots$, $g_{n-k+l}$ are complex-analytic functions of $w_{1}, \cdots, w_{l}$. An argument similar to the previous one gives us that the Levi form on $M$ vanishes at $p$ if and only if the complex Hessians at $p$ of each of the functions $h_{1}, \cdots, h_{k-2 l}$ with respect to the variables $w_{1}, \cdots, w_{l}$ are all zero matrices.

4. Certain open sets. We wish to show that to discuss local holomorphicity we need only consider open sets with $\mathscr{C}^{\infty}$ boundaries.

Suppose our manifold $M$ is locally $C-R$ at $p \in M$ and $\Omega$ is a pseudoconvex open set of $C^{n}$ containing a compact neighborhood $N$ of $p$ in $M$. Let $K$ be a compact subset of $\Omega$ also containing $N$, and let $W$ be an open neighborhood of $\hat{K}_{\Omega, P}$, the plurisubharmonic hull of $K$ in $\Omega$. Since $\hat{K}_{\Omega, P}$ is compact in $\Omega$, we may take $W \subset \subset \Omega$, where $\subset \subset$ denotes relative compactness. We need the following theorem [2].

Theorem 1. Let $\Omega, K$, and $W$ be as stated. Then there exists a function $u \in \mathscr{C}^{\infty}(\Omega)$ such that

(a) $u$ is strictly plurisubharmonic for every $z \in \Omega$.

(b) $u<0$ in $K$ but $u>0$ in $\Omega \cap\{$ complement of $W\}$.

(c) $\{z, z \in \Omega, u(z)<c\} \subset \subset \Omega$ for every $C \in \boldsymbol{R}$.

Since $\bar{W}$ is a compact subset of $\Omega$ and $u$ is a continuous realvalued function on $\Omega$, there exists a real number $c^{\prime} \geqq 0$ such that $u \leqq c^{\prime}$ in $\bar{W}$. Letting $\Omega^{\prime}=\left\{z ; z \in \Omega ; u(z)<c^{\prime}\right\}$, we have that $\Omega^{\prime} \subset \subset \Omega$ and $\partial Q^{\prime}=\left\{z ; z \in \Omega ; u(z)=c^{\prime}\right\}$. Thus $\Omega^{\prime}$ is an open manifold in $C^{n}$ such that

(i) $\Omega^{\prime}$ contains $N$,

(ii) $\Omega^{\prime}$ has a $\mathscr{C}^{\infty}$ boundary,

(iii) $\bar{\Omega}^{\prime}$ is a compact subset of $\Omega$, and

(iv) the function defining $\partial \Omega^{\prime}$ has a positive definite Hessian at every point of $\partial \Omega^{\prime}$.

We shall restrict ourselves to the case $k>n$ since discussions for $k \leqq n$ follow in an analogous manner. Thus $M$ has local equations (1) near $p$. Let us assume $L_{p}(M) \neq 0$, and arbitrarily that $\partial^{2} h_{1} / \partial w_{1} \partial \bar{w}_{1}(p)=-1$ with the Hessian of $h_{1}$ in diagonal form. Coordinate changes on $C^{n}$ (see [2], p. 51) allow us to write

$$
y_{1}-h_{1}(z)=y_{1}-\sum_{j=1}^{n-l} \frac{\partial^{2} h_{1}}{\partial z_{j} \partial \bar{z}_{j}}(p) z_{i} \bar{z}_{j}+0\left(|z|^{3}\right) \text {. }
$$


Using continuity arguments and properties of superharmonic functions in the variable $w_{1}$ we find there exists a closed neighborhood $A$ of $p$ in $M$ such that $h_{1}$, as a function of $w_{1}, \bar{w}_{1}$ with the other variables fixed, cannot assume a relative minimum at any interior point of $A$. Thus for fixed $x_{1}, \cdots, x_{2(n-l)-k}, w_{2}, \cdots, w_{k-n+l}$, the minimum of $h_{1}$ as a function of $w_{1}, \bar{w}_{1}$ is attained on the boundary of $A$. We may assume this minimum is $\leqq-\varepsilon, \varepsilon>0$, for every $x_{1}, \cdots$, $x_{2(n-l)-k}, w_{2}, \cdots, w_{k-n+l}$ with $\left(x_{1}, \cdots, x_{2(n-l)-k}, w_{1}, \cdots, w_{k-n+l}\right) \in A$.

We define a new set $S$ with local equations at $p$ given by

$$
\begin{aligned}
& z_{1}=x_{1}+i y_{1} \\
& z_{2}=x_{2} \\
& \vdots \\
& z_{2(n-l)-k}=x_{2(n-l)-k} \\
& z_{2(n-l)-k+1}=u_{1}+i v_{1}=w_{1} \\
& \vdots \\
& z_{n-l}=u_{k-n+l}+i v_{k-n+l}=w_{k-n+l} \\
& z_{n-l+1}=0 \\
& \vdots \\
& z_{n}=0
\end{aligned}
$$

where $-\varepsilon \leqq y_{1} \leqq h_{1}\left(x_{1}, \cdots, x_{2(n-l)-k}, w_{1}, \cdots, w_{k-n+l}\right)$, and where $\left(x_{1}, \cdots\right.$, $\left.x_{2(n-l)-k}, w_{1}, \cdots, w_{k-n+l}\right)$ are in $A$.

Denote the projection of $C^{n}$ onto the set

$$
\left\{z \in C^{n} ; y_{2}=0, \cdots, y_{2(n-l)-k}=0, z_{n-l+1}=0, \cdots, z_{n}=0\right\} \text { by } \pi \text {. }
$$

Under this projection $A$ goes onto the subset of $S$ with $y_{1}=h_{1}\left(x_{1}, \cdots\right.$, $\left.x_{2(n-l)-k}, w_{1}, \cdots, w_{k-n+l}\right)$.

Let $\left\{\Omega_{i}\right\}_{i=1}^{\infty}$ be domains of holomorphy in $C^{n}$ such that $M$ is locally holomorphic at $p=0$ with respect to these domains, and each contains $A$. Denote by $\left\{\Omega_{i}^{\prime}\right\}_{i=1}^{\infty}$ the sets described in (3), and let $u_{i}^{\prime}$ be the $C^{\infty}$ defining function for $\partial \Omega_{i}^{\prime}$. As $i$ gets large $\partial \Omega_{i}^{\prime}$ tends to $M$, so there exists an integer $i$ and a nonempty open set $U$ of points in $S$ such that $\pi\left(\partial \Omega_{i}^{\prime}\right)$ does not contain $U$. Since $\partial \Omega_{i}^{\prime}$ cannot intersect $M$ there exists a point $q \in \Omega_{i}^{\prime}$ such that $\pi(q) \in S-\pi(A), \partial u_{i}^{\prime} / \partial y_{1}^{\prime}(q) \neq 0$, and $y_{1}$ as a function of $w_{1}, \bar{w}_{1}$ (with $x_{1}, z_{2}, \cdots, z_{2(n-l)+k^{\prime}}, w_{2}, \cdots, w_{k-n+l}, z_{n-l+1}, \cdots, z_{n}$ held fixed) has a relative maximum at $q$. However $y_{1}$ is a (strictly) subharmonic function of $w_{1}, \bar{w}_{1}$ since $u_{i}^{\prime}$ is and we have our contradiction. Geometrically we have, for fixed $x_{1}, z_{2}, \cdots, z_{2(n-l)+k}, w_{2}, \cdots, w_{k-n+l}$, $z_{n-l+1}, \cdots, z_{n}$, a real 2-dimensional manifold (with coordinates $w_{1}, \bar{w}_{1}$ ) which lies in or below the tangent plane to $\partial \Omega_{i}^{\prime}$ at $q$. This implies the 
complex Hessian of $u_{i}^{\prime}$ at $q$ has a nonpositive eigenvalue, also a contradiction.

We have the following result, the sufficiency being found in [6].

THeOREM 2. Let $M$ be a real $k$-dimensional $\mathscr{C}^{2}$ submanifold of $C^{n}$ which is locally $C-R$ at $p \in M$ with complex dimension $H_{p}(M)=$ $m>0$. Then $M$ is locally holomorphic at $p$ if and only if the Levi form vanishes identically near $p$.

5. Odd codimensional submanifolds. Suppose $M$ is a real $(2 n-2 m+1)$-dimensional $\mathscr{C}^{\infty}$ submanifold of $C^{n}$, where $m$ is a positive integer and $(2 n-2 m+1) \geqq 2$.

Definition 1. The manifold $M$ is a local pseudo-hypersurface at $p \in M$ if $M$ is locally $C-R$ at $p \in M$ and $p$ is an exceptional point of the highest possible order. The manifold $M$ is a pseudo-hypersurface if it is a local pseudo-hypersurface at every one of its points.

The reasons for the name pseudo-hypersurface are the following:

(i) In the local equations for $M$ at a point $p$ there is only the function $h_{1}$ in (1), and if $M$ is indeed real-analytic, coordinate changes can be made so that $M$ appears locally as a hypersurface in some possibly lower dimensional complex Euclidean space,

(ii) if the Levi form on $M$ does not vanish at $p$, we shall show that all $C-R$ functions on $M$ extend to holomorphic functions on some open set in $C^{n}$, similar to the hypersurface case.

Let $M$ be a real $k$-dimensional $\mathscr{C}^{\infty}$ manifold embedded in $C^{n}, k$, $n \geqq 2$. Suppose $f \in \mathscr{C}^{\infty}(M)$. We say that $f$ is a $C-R$ function at $p \in M$ if $\bar{X} f(y)=0$, for $y$ near $p$ and $X$ any section of $H(M)$. If $M$ is locally $C-R$ at $p$ it suffices to verify the equality just for $X$ in a local basis for $H(M)$ at $p$. We note that our manifold need not be globally $C-R$. Thus we may have points which are not locally $C-R$, but obviously the set of such points is nowhere dense in $M$. The function $f$ is a $C-R$ function on $M$ if $f$ is a $C-R$ function at each point of $M$. The $C-R$ functions are denoted by $C R(M)$.

We say that $M$ is $C-R$ extendible to a connected set $K=M U$ $K^{\prime}$ where $K^{\prime} \neq \phi$, if for every $f \in C R(M)$ there exists a continuous $F: M \cup K^{\prime} \rightarrow C$ so that $F \mid M=f$ and $F \mid K^{\prime} \in \mathcal{O}\left(K^{\prime}\right)$. (By $\mathcal{O}\left(K^{\prime}\right)$ we denote those functions holomorphic in some open neighborhood of $K^{\prime}$ ).

If $M$ is a local pseudo-hypersurface at $p \in M$, the local equations of $M$ in an open neighbhorhood of our point $p$ are 


$$
\begin{aligned}
& z_{1}=x_{1}+i h_{1}\left(x_{1}, w_{1}, \cdots, w_{n-m}\right) \\
& z_{2}=u_{1}+i v_{1}=w_{1} \\
& \vdots \\
& z_{n-m+1}=u_{n-m}+i v_{n-m}=w_{n-m} \\
& z_{n-m+z}=g_{1}\left(x_{1}, w_{1}, \cdots, w_{n-m}\right) \\
& \vdots \\
& z_{n}=g_{m-1}\left(x_{1}, w_{1}, \cdots, w_{n-m}\right) .
\end{aligned}
$$

We have by Lemma 2.6.2 of [4] that every function which is $C-R$ in an open neighborhood of $p$ in $M$ is also $C-R$ in an open neighborhood of $p$ in the hypersurface with local equations at $p$

$$
\begin{aligned}
& z_{1}=x_{1}+i h_{1}\left(x_{1}, w_{1}, \cdots, w_{n-m}\right) \\
& z_{2}=u_{1}+i v_{1}=w_{1} \\
& \vdots \\
& z_{n}=u_{n-1}+i v_{n-1}=w_{n-1} .
\end{aligned}
$$

However this is a hypersurface which is $C-R$ extendible over an open subset of $\boldsymbol{C}^{n}$ if the Levi form is nonvanishing at $p$ (see Theorem 2.6.13 in [2]). We have shown the following result.

THEOREM 3. Suppose $M$ is a local pseudo-hypersurface at $p \in M$ and the Levi form on $M$ is nonvanishing at $p$. Then $M$ is $C-R$ extendible over an open subset of $\boldsymbol{C}^{n}$.

REMARK 1. Nirenberg [4] proves a much more general theorem (in the sense of dimensions and genericity) than the one above, but requires in the pseudo-hypersurface case that the complex Hessian of $h_{1}$ with respect to $w_{1}, \cdots, w_{n-m}$ have either two eigenvalues of opposite signs or all nonzero eigenvalues of the same sign. It is hoped that $C-R$ extendibility theorems depend only on the vanishing or nonvanishing of the Levi form.

REMARK 2. Since a compact pseudo-hypersurface has a peak point where the Levi form does not vanish (see [3]), it is $C-R$ extendible over an open set in $\boldsymbol{C}^{n}$.

REMARK 3. This is the first example of a lower dimensional extendibility theorem without using the work of Bishop.

\section{REFERENCES}

1. S. J. Greenfield, Cauchy-Riemann equations in several variables, Annali Scuola Norm. Sup. di Pisa, 22 (1968), 275-314. 
2. L. Hormander, An introduction to complex analysis in several variables, Van Nostrand, Princeton, N. J., 1966.

3. L. R. Hunt, Real-analytic submanifolds of complex manifolds, Proc. Amer, Math. Soc., 29 (1971), 69-74.

4. R. Nirenberg, On the H. Lewy extension phenomenon, Trans. Amer. Math. Soc., (1972), 337-356.

5. R. Nirenberg and R. O. Wells, Jr., Approximation theorems on differentiable submanifolds of a complex manifold, Trans. Amer. Math. Soc., 142 (1969), 15-35.

6. R. O. Wells, Jr., Holomorphic hulls and holomorphic convexity of differentiable submanifolds, Trans. Amer. Math. Soc., 132 (1968), 245-262.

7. - Concerning the envelope of holomorphy of a compact differentiable submanifold of a complex manifold, Annali Scuola Norm Sup. di Pisa, 23 (1969), 347-361.

Received May 7, 1971 and in revised form October 29, 1971.

TeXas TeCh University 


\section{PACIFIC JOURNAL OF MATHEMATICS}

\section{EDITORS}

\section{H. SAMELSON}

Stanford University

Stanford, California 94305

\section{R. HOBBY}

University of Washington

Seattle, Washington 98105

\section{J. DugundjI}

Department of Mathematics University of Southern California Los Angeles, California 90007

RICHARD ARENS

University of California

Los Angeles, California 90024

\section{ASSOCIATE EDITORS}
E. F. BECKENBACH
B. H. NeumanN
F. WOLF
K. YOSHIDA

\section{SUPPORTING INSTITUTIONS}

\author{
UNIVERSITY OF BRITISH COLUMBIA \\ CALIFORNIA INSTITUTE OF TECHNOLOGY \\ UNIVERSITY OF CALIFORNIA \\ MONTANA STATE UNIVERSITY \\ UNIVERSITY OF NEVADA \\ NEW MEXICO STATE UNIVERSITY \\ OREGON STATE UNIVERSITY \\ UNIVERSITY OF OREGON \\ OSAKA UNIVERSITY
}

\author{
UNIVERSITY OF SOUTHERN CALIFORNIA \\ STANFORD UNIVERSITY \\ UNIVERSITY OF TOKYO \\ UNIVERSITY OF UTAH \\ WASHINGTON STATE UNIVERSITY \\ UNIVERSITY OF WASHINGTON \\ AMERICAN MATHEMATICAL SOCIETY \\ NAVAL WEAPONS CENTER
}

The Supporting Institutions listed above contribute to the cost of publication of this Journal, but they are not owners or publishers and have no responsibility for its content or policies.

Mathematical papers intended for publication in the Pacific Journal of Mathematics should be in typed form or offset-reproduced, (not dittoed), double spaced with large margins. Underline Greek letters in red, German in green, and script in blue. The first paragraph or two must be capable of being used separately as a synopsis of the entire paper. The editorial "we" must not be used in the synopsis, and items of the bibliography should not be cited there unless absolutely necessary, in which case they must be identified by author and Journal, rather than by item number. Manuscripts, in duplicate if possible, may be sent to any one of the four editors. Please classify according to the scheme of Math. Rev. Index to Vol. 39. All other communications to the editors should be addressed to the managing editor, Richard Arens, University of California, Los Angeles, California, 90024.

50 reprints are provided free for each article; additional copies may be obtained at cost in multiples of 50 .

The Pacific Journal of Mathematics is published monthly. Effective with Volume 16 the price per volume (3 numbers) is $\$ 8.00$; single issues, $\$ 3.00$. Special price for current issues to individual faculty members of supporting institutions and to individual members of the American Mathematical Society: $\$ 4.00$ per volume; single issues $\$ 1.50$. Back numbers are available.

Subscriptions, orders for back numbers, and changes of address should be sent to Pacific Journal of Mathematics, 103 Highland Boulevard, Berkeley, California, 94708.

PUBLISHED BY PACIFIC JOURNAL OF MATHEMATICS, A NON-PROFIT CORPORATION

Printed at Kokusai Bunken Insatsusha (International Academic Printing Co., Ltd.), 270, 3-chome Totsuka-cho, Shinjuku-ku, Tokyo 160, Japan. 


\section{Pacific Journal of Mathematics}

\section{Vol. 42, No. $3 \quad$ March, 1972}

Catherine Bandle, Extensions of an inequality by Pólya and Schiffer for vibrating membranes ................................ 543

S. J. Bernau, Topologies on structure spaces of lattice groups.......... 557

Woodrow Wilson Bledsoe and Charles Edward Wilks, On Borel product measures .......................................

Eggert Briem and Murali Rao, Normpreserving extensions in subspaces of

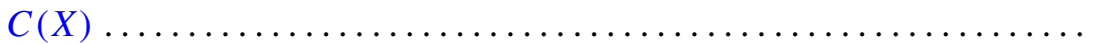

Alan Seymour Cover, Generalized continuation.................. 589

Larry Jean Cummings, Transformations of symmetric tensors .......... 603

Peter Michael Curran, Cohomology of finitely presented groups .......... 615

James B. Derr and N. P. Mukherjee, Generalized quasicenter and

hyperquasicenter of a finite group ...................... 621

Erik Maurice Ellentuck, Universal cosimple isols .................. 629

Benny Dan Evans, Boundary respecting maps of 3-mainfolds .......... 639

David F. Fraser, A probabilistic method for the rate of convergence to the

Dirichlet problem .................................. 657

Raymond Taylor Hoobler, Cohomology in the finite topology and Brauer

groups ..................................... 667

Louis Roberts Hunt, Locally holomorphic sets and the Levi form ........ 681

B. T. Y. Kwee, On absolute de la Vallée Poussin summability............ 689

Gérard Lallement, On nilpotency and residual finiteness in semigroups .... 693

George Edward Lang, Evaluation subgroups of factor spaces........... 701

Andy R. Magid, A separably closed ring with nonzero torsion pic ....... 711

Billy E. Rhoades, Commutants of some Hausdorff matrices ............. 715

Maxwell Alexander Rosenlicht, Canonical forms for local derivations . . . . 721

Cedric Felix Schubert, On a conjecture of L. B. Page ................ 733

Reinhard Schultz, Composition constructions on diffeomorphisms of $S^{p} \times S^{q}$

J. P. Singhal and H. M. (Hari Mohan) Srivastava, A class of bilateral generating functions for certain classical polynomials ....

Richard Alan Slocum, Using brick partitionings to establish conditions which insure that a Peano continuum is a 2-cell, a 2-sphere or an annulus...

James F. Smith, The p-classes of an $H^{*}$-algebra ...

Jack Williamson, Meromorphic functions with negative zeros and positive

poles and a theorem of Teichmuller ................. 